\title{
Preparation and physicochemical characterization of meloxicam orally fast disintegration tablet using its solid dispersion
}

\author{
Zahra Shoormeij $^{1}$, Azade Taheri ${ }^{1 *}$, Alireza Homayouni ${ }^{1}$ \\ ${ }^{1}$ Novel Drug Delivery Systems Research Center, Department of Pharmaceutics, Faculty of Pharmacy, Isfahan University of \\ Medical sciences, Isfahan, Iran
}

\begin{abstract}
Meloxicam (MLX) is a non-steroidal, anti-inflammatory drug that is prescribed in the treatment of rheumatoid arthritis and osteoarthritis. MLX is practically insoluble in water and exhibits a slow onset of action. In this study, MLX solid dispersions (MLX SDs) were prepared to improve the water solubility of this poorly water-soluble drug. Then orally disintegrating tablets (ODT) of MLX were developed using MLX SD to decrease the onset of action of this drug. MLX, poloxamer 188, and crospovidone of different ratios were melted in molten poloxamer 188 as a hydrophilic carrier. The optimum SD with the highest saturation solubility in water (13.09 $\pm 0.34 \mathrm{microgram} / \mathrm{mL})$ consisting of MLX: poloxamer 188: crospovidone in the ratio of 1:2:0 was used for the preparation of MLX ODTs. MLX ODTs were prepared by the direct compression method and optimized by the $2^{3}$ factorial design. The effect of the superdisintegrant concentration, the mannitol-avicel ratio, and the level of compression force on the disintegration time, hardness, and percent of dissolved MLX from MLX ODTs after 30 min was evaluated. DSC and XRD analysis approved an amorphous form of MLX in SDs. The optimized ODT formulation containing $10 \%$ of superdisintegrant, and mannitol and avicel in the ratio of 4:1 respectively was compressed using a high level of compression force. The optimized ODT showed hardness ( $34.37 \pm 2.1$ $\mathrm{N})$ and friability $(1.26 \pm 0.04 \%)$. This formulation showed a rapid disintegration in $12.66 \pm 2.5$ seconds, which $82.66 \pm 5.1 \%$ of the MLX released within $30 \mathrm{~min}$. MLX ODTs, prepared from MLX SD, could be introduced as a suitable dosage form of MLX with improved solubility and the onset of action.
\end{abstract}

Keywords: Meloxicam/physicochemical characterization. Meloxicam/onset of action. Solubility.

\section{INTRODUCTION}

Meloxicam (MLX) is a non-steroidal antiinflammatory drug that is prescribed in the treatment of rheumatoid arthritis and osteoarthritis with low gastrointestinal complications (Pairet et al., 1998; Distel et al., 1996; Ahmed, Khanna, Furst, 2005; Aoki et al., 2006). MLX is practically insoluble in water (belonging to BCS Class II) and exhibits a slow oral absorption and slow onset of action (Budavari et al., 2001; Ghorab et al., 2004). A rapid onset of action is important for patients, particularly under the pain condition of rheumatism and osteoarthritis. Consequently, a suitable oral dosage form of MLX, with enhanced aqueous solubility, could facilitate

\footnotetext{
* Correspondence: A. Taheri. Department of Pharmaceutics, Faculty of Pharmacy and Novel Drug Delivery Systems Research Center, Isfahan University of Medical sciences, Postal Code 81746-73461 - Isfahan, Iran. Tel: +98 3137927114 / Fax: +98 31 36692288. E-mail: az.taheri@pharm.mui.ac.ir
}

its oral absorption and reduce its onset of action for the treatment of acute pain. Different methods such as salt formation (Serajuddin, 2007), emulsification ( $\mathrm{He}, \mathrm{He}$, Gao, 2010), and nano-pulverization (Merisko-Liversidge, Liversidge, Cooper, 2013) had been used to improve the solubility and bioavailability of poorly soluble drugs. The preparation of solid dispersion (SD) is a feasible and effective method to improve the dissolution bioavailability of poorly water-soluble drugs including MLX (Leuner, Dressman, 2000). SDs are dispersions of one or more active pharmaceutical ingredients (API) in an inert carrier or matrix such as biodegradable polymers in solid state, prepared by melting (fusion), solvent, or a melting-solvent method (Chiou, Riegelman, 1971). It has been suggested that orally disintegrating tablets (ODT) have a faster onset of action compared to conventional tablets, and are more appropriate for the treatment of acute pain (Rauck et al., 2009). In addition, ODTs could reduce dysphagia in older patients suffering from arthritis (Shen et al., 2007). With 
these in mind, the objective of this study was to enhance the solubility of MLX through the preparation of its SDs by the melting method and then decrease its onset of action by the preparation of ODTs from the prepared solid dispersion of this poorly water-soluble drug.

\section{MATERIAL AND METHODS}

\section{Material}

Poloxamer188 was supplied by BASF (Germany). Crospovidone was purchased from Sigma-Aldrich (Germany). Meloxicam (MLX), mannitol, microcrystalline cellulose (Avicel PH-101), aspartame and magnesium stearate were purchased from the Farabi Pharmaceutical Company (Iran). All other solvents and chemicals were of an analytical grade.

\section{Preparation of SD of meloxicam with poloxamer 188}

Accurately weighed quantities of MLX and crospovidone in different ratios were dissolved in melted poloxamer 188 and mixed carefully to obtain a homogenous mixture. The amount of MLX, crospovidone and poloxamer188 utilized for the preparation of MLX solid dispersions (MLX SD) are mentioned in Table I. After the preparation of homogenous mixtures, the prepared mixtures were immediately cooled in ice.

\section{Preparation of physical mixtures of MLX and carriers}

The physical mixtures were prepared by weighing the calculated amounts of MLX, poloxamer 188, and crospovidone in the same ratio of solid dispersion samples and mixing them in a mortar by triturating. The resultant physical mixtures were passed through a 16-mesh sieve.

\section{Characterization of SD}

\section{Drug content determination in SD samples}

An accurately weighed amount of each solid dispersion sample was dissolved in $5 \mathrm{~mL}$ of methanol and, then, $1 \mathrm{~mL}$ of $0.1 \mathrm{~N} \mathrm{NaOH}$ was added. A phosphate buffer of $\mathrm{pH} 7.5$ was added until the volume reached $100 \mathrm{~mL}$. The resulting suspension was filtered through a $0.22-\mu \mathrm{m}$ filter. The concentration of MLX was determined spectrophotometrically at $362 \mathrm{~nm}$ (UV mini/420 Shimadzo, Japan). The concentration of each sample was determined in triplicates and the mean value and standard deviation were reported.

\section{Determination of saturation solubility}

Saturation solubility studies were performed on pure MLX, physical mixtures, and SD samples. An excess amount of the samples was added to a volumetric flask containing $20 \mathrm{~mL}$ of distilled water and stirred at $100 \mathrm{rpm}$ for $48 \mathrm{~h}$ at $25{ }^{\circ} \mathrm{C}$. The resulting suspension was filtered through a $0.22-\mu \mathrm{m}$ filter. The concentration of MLX was determined spectrophotometrically at $362 \mathrm{~nm}$. The solubility of each sample was determined in triplicates and the mean value and standard deviation were reported.

\section{Differential scanning calorimetry (DSC) analysis}

The thermal behaviors of MLX, poloxamer 188,

TABLE I - The amount of MLX, poloxamer 188 and crospovidone that utilized for preparation of MLX SDs and the characteristics of prepared MLX SDs

\begin{tabular}{lccccccc}
\hline Formulation & MLX & Poloxamer & $\begin{array}{c}\text { MLX/ } \\
\text { poloxamer } \\
\mathbf{1 8 8} \text { ratio }\end{array}$ & Crospovidone & $\begin{array}{c}\text { Saturation } \\
\text { solubility } \\
(\boldsymbol{\mu g} / \mathbf{m l})\end{array}$ & $\begin{array}{c}\text { Saturation } \\
\text { Drug content } \\
\text { solubility } \\
\text { of physical } \\
\text { mixture } \\
(\boldsymbol{\mu g} / \mathbf{m l})\end{array}$ \\
\hline SD1 & $200 \mathrm{mg}$ & $200 \mathrm{mg}$ & 1 & ----- & $10.41 \pm 1.71$ & $82.7 \pm 2.36$ & $8.43 \pm 1.142$ \\
$\mathrm{SD} 2$ & $200 \mathrm{mg}$ & $400 \mathrm{mg}$ & 2 & ----- & $13.09 \pm 0.34$ & $92.4 \pm 3.14$ & $9.01 \pm 0.345$ \\
$\mathrm{SD} 3$ & $200 \mathrm{mg}$ & $800 \mathrm{mg}$ & 4 & ---- & $12.1 \pm 1.19$ & $94.1 \pm 3.16$ & $9.17 \pm 0.239$ \\
$\mathrm{SD} 4$ & $200 \mathrm{mg}$ & $200 \mathrm{mg}$ & 1 & $200 \mathrm{mg}$ & $2.6 \pm 0.011$ & $74.8 \pm 4.25$ & $7.1 \pm 0.471$ \\
$\mathrm{SD} 5$ & $200 \mathrm{mg}$ & $400 \mathrm{mg}$ & 2 & $200 \mathrm{mg}$ & $3.1 \pm 0.38$ & $84.1 \pm 2.85$ & $8.6 \pm 0.609$ \\
SD6 & $200 \mathrm{mg}$ & $800 \mathrm{mg}$ & 4 & $200 \mathrm{mg}$ & $4.3 \pm 0.305$ & $82.5 \pm 2.45$ & $8.79 \pm 0.61$ \\
\hline
\end{tabular}


crospovidone, selected sample of SDs with higher saturation solubility and their physical mixture were analyzed using NETZSCH DSC 200 F3 instrument (NETZSCH, Germany). Five milligrams of samples were placed in aluminum pans. The samples were heated from 20 to $280^{\circ} \mathrm{C}$ at a scanning rate of $10^{\circ} \mathrm{C} / \mathrm{min}$. The melting point and enthalpy of fusion were automatically calculated using the software provided.

\section{XRD Analysis}

The crystallinity of MLX, poloxamer188, crospovidone, and the selected samples of SDs with higher saturation solubility and their physical mixture were evaluated with an X-ray diffractometer (Philips X'PERT, Germany). The diffraction patterns were measured with a voltage of $40 \mathrm{kV}$ and a current of $30 \mathrm{~mA}$. X-ray diffraction data were collected at room temperature in the range of $10^{\circ}<2 \theta<40^{\circ}$.

\section{Experimental design}

A $2^{3}$ full-factorial experimental design was applied to determine the optimum condition for the preparation of MLX orally disintegrating tablets (MLX ODTs) using the Design-Expert 7 software (State Ease. Inc., Minneapolis, MN, USA). The percent of crospovidone as a superdisintegrant (X1), the mannitol-avicel ratio (X2), and the compression force applied for tablet compression (X3) were selected as independent variables. Each factor was set at high and low levels. The actual values of different variables are given in Table II. Eight formulations of MLX ODTs (F1 to F8) were prepared according to the design as shown in Table II. The hardness, disintegration time, and the percent of meloxicam that dissolved in the dissolution medium after $30 \mathrm{~min}$. were taken as response parameters.

TABLE II - The actual values of different independent variables that used for determination of the optimum conditions for preparation of MLX orally disintegrating tablets

\begin{tabular}{lcc}
\hline Variable & High Level & Low level \\
\hline Percent of disintegrant & $10 \%$ & $5 \%$ \\
Mannitol/avicel ratio & 4 & 2 \\
Compression force & High & low \\
\hline
\end{tabular}

\section{Preparation of orally disintegrating tablets (ODT)}

ODT of MLX was prepared by the direct compression method. All ingredients were separately passed through a 60-mesh sieve. MLX SD, mannitol, avicel, crospovidone (as superdisintegrant) and aspartame (as sweetener) were accurately weighed and properly mixed up. The blends were lubricated with $1 \%$ magnesium stearate. An evaluation of the mixed blends of MLX SD and excipients were carried out for angle of repose, bulk density, tapped density, compressibility and flowability (Lieberman, Lachman, 1989).

The tablets were directly compressed using a singlepunch tableting machine (Erweka AR 4100, Germany) equipped with a 6-mm round flat punch. The tablets were compressed to a target weight of $150 \mathrm{mg}$.

\section{Characterization of MLX ODTs}

\section{Drug content determination in tablets}

Ten tablets from each batch were weighed separately. Each tablet was accurately weighed, dissolved in $5 \mathrm{~mL}$ of methanol and $1 \mathrm{~mL}$ of $0.1 \mathrm{~N} \mathrm{NaOH}$ and the volume was made up to $100 \mathrm{~mL}$ with PBS (pH 7.5) and stirred until MLX was dissolved completely. The resulting suspension was filtered through a 0.22 $\mu \mathrm{m}$ filter. The concentration of MLX was determined spectrophotometrically at $362 \mathrm{~nm}$. The result of each sample was determined in triplicate and the mean value and standard deviation were reported.

\section{Determination of friability}

A friability analysis was performed on 20 tablets at a speed of $25 \mathrm{rpm}$ with tablets dropping from a height of six inches for 100 revolutions using a Roche type friabilitor (Erweka, TB-24, Germany). The accurate weights of the tablets were determined before and after the test. The friability was measured as a percentage of weight lost during a standardized abrasion.

\section{Tablet disintegration time}

The in vitro disintegration time of MLX ODTs was determined using an Erweka ZT (Germany) disintegration apparatus with $1000 \mathrm{~mL}$ of purified water, maintained at $37 \pm 0.5^{\circ} \mathrm{C}$. The disintegration time was recorded once the tablets were completely disintegrated and passed through the mesh. Six tablets per formulation were tested and the mean value and standard deviation reported.

\section{Tablet hardness}

The hardness of MLX ODTs was evaluated by individually measuring the breaking strength in the radial 
direction of 8 tablets using a tablet hardness tester with an Erweka TBH 30 MD equipment (Germany).

\section{Dissolution test}

The release rate of MLX from MLX ODTs ( $n=3$ ) was determined using a USP apparatus Type II at 75 rpm (Electro lab, TDT-08L). The dissolution test was performed using $900 \mathrm{~mL}$ of phosphate buffer $\mathrm{pH} 7.5$ as a dissolution medium, at $37 \pm 0.5^{\circ} \mathrm{C}$. A sample $(2 \mathrm{~mL})$ of the dissolution medium was withdrawn from the dissolution vessels at 2, 5, 10, 15, 20 and 30 min and replaced with a fresh dissolution medium. The samples were filtered through a $0.22-\mu \mathrm{m}$ membrane filter. The absorbance of these solutions was measured at $362 \mathrm{~nm}$ using a Shimadzu spectrophotometer. The cumulative percentage of the release of MLX was calculated using an equation obtained from a standard curve.

\section{RESULTS AND DISCUSSION}

\section{Saturation solubility of MLX SDs and physical mixtures}

The results of the solubility studies indicated that pure MLX had a very low solubility in water at $25^{\circ} \mathrm{C}(6.58 \pm 0.21 \mu \mathrm{g} / \mathrm{mL})$. The saturation solubility of MLX increased in the presence of poloxamer 188 and crospovidone in the physical mixtures. An increase in the concentration of poloxamer 188 in the physical mixture samples increased the saturation solubility of MLX. This phenomenon could be attributed to an improvement of the MLX wettability because of high water solubility of poloxamer 188 (Jung et al., 1999). As shown in Table I, MLX SDs that contain MLX and poloxamer 188 (SD1, SD2 and SD3) show a significant increase in saturation solubility compared to the pure MLX and physical mixtures with the same ratios ( $p$ value $<0.05$ ). In SD1 and SD2, the saturation solubility of MLX increased after the amount of poloxamer 188 was raised. This was because of the high water solubility of poloxamer 188 (Jung et al., 1999). The preparation of the dosage form of solid dispersions (SDs) is a critical aspect that should be considered by scientists. The pulverization of the solid dispersions (SDs) prepared by the melting method is difficult. In addition, these SDs are often soft and tacky and have poor flow characteristics. Consequently, the addition of more excipients to the SDs is often necessary (Serajuddin, 1999; Leuner, Dressman, 2000; Shibata et al., 2009). These difficulties can be overcome by the addition of crospovidone, as a carrier, to SDs (Fujii et al, 2005;
Shibata et al., 2009). However, crospovidone does not melt or dissolve in melted poloxamer but crospovidone possesses several useful characteristics that can improve the SD proprieties when added to them. Firstly, a good flow property and compressibility of crospovidone can improve the fluidity and tableting ability of the SD powder (Fujii et al., 2005). Secondly, the same chemical structure of crospovidone and polyvinylpyrrolidone (PVP), which is commonly used as a carrier of SDs (Leuner, Dressman, 2000), can improve the water solubility of poorly soluble drugs in SDs.

In this study, crospovidone was added to three formulations of MLX SDs to improve their flow and compressibility properties. But the saturation solubility of MLX SD samples that contained crospovidone (SD4, SD5, and SD6) was lower than the values predicted, according to the saturation solubility of the physical mixtures having the same ratios (Table I). The lower saturation solubility of MLX SDs that contained crospovidone (SD4, SD5, and SD6) compared to the predicted value may be related to a decrease in the water sorption of MLX SDs due to MLX-crospovidone interaction in the solid dispersion. The interaction could decrease the number of available polar groups that could interact with water during the water sorption and dissolution phenomena. Moreover, the formation of the gel formed by crospovidone around the solid dispersions could increase the viscosity in the donor phase and decrease the saturation solubility of MLX (Jung et al., 1999; Punčochová et al., 2014).

As shown in Table 1, batch number SD2 (containing MLX: poloxamer 188: crospovidone in the ratio 1:2:0) exhibited the maximum saturation solubility equal to $13.09 \pm 0.347 \mu \mathrm{g} / \mathrm{mL}$ and was used for the preparation of MLX ODTs.

\section{Drug content of MLX SDs}

The prepared MLX SDs showed excellent entrapment of MLX. The MLX content in the solid dispersions was found to be in the range of $74.8 \pm 4.25$ to $94.1 \pm 3.16 \%$ as shown in Table I. Batch SD3 formulation (containing MLX: poloxamer 188: crospovidone in the ratio of $1: 4: 0)$ showed the highest drug content of $94.1 \pm 3.16 \%$, while batch SD4 (containing MLX: poloxamer 188: crospovidone in the ratio of $1: 1: 1)$ showed the least drug content of $74.8 \pm 4.25 \%$.

\section{Differential scanning calorimetry (DSC) analysis}

A DSC analysis was performed in order to screen the solid-state properties of solid dispersions (SDs) and 
physical mixture samples. This analysis could be used to evaluate any interaction between MLX, poloxamer 188, and crospovidone. The DSC thermograms of poloxamer 188, crospovidone, pure MLX, SD2 (MLX: poloxamer 188: crospovidone ratio=1:2:0), SD5 (MLX: poloxamer 188: crospovidone ratio=1:2:1) and MLX, poloxamer 188, and crospovidone physical mixture have been depicted in Figure 1. MLX showed a sharp endothermic peak at 262.5 ${ }^{\circ} \mathrm{C}$, indicating its melting points. Poloxamer 188 showed a sharp endothermic peak at $60.6^{\circ} \mathrm{C}$, indicating its melting. The DSC thermogram of physical mixture showed two endothermic peaks at $55.4^{\circ} \mathrm{C}$ and $250.5^{\circ} \mathrm{C}$, indicating the melting points of poloxamer 188 and MLX respectively. The presence of polymer in the physical mixture sample decreased the melting temperature of MLX and its fusion enthalpy, perhaps due to the miscibility of drug/polymer, thus resulting in a partial dissolution of MLX in polymers during the heating process (Jahangiri et al., 2015; Shah et al., 2007). Furthermore, these reductions in the melting point and enthalpy of fusion were greater for solid dispersion samples (SD2 and SD5). The melting temperature of MLX and enthalpy of fusion were $250.5^{\circ} \mathrm{C}$ and $\Delta \mathrm{H}=422 \mathrm{~J} / \mathrm{g}$ for physical mixture, while these records were $209.6{ }^{\circ} \mathrm{C}$ and $\Delta \mathrm{H}=97.98 \mathrm{~J} / \mathrm{g}$ for $\mathrm{SD} 2$ and $207.3^{\circ} \mathrm{C}$ and $\Delta \mathrm{H}=209.5 \mathrm{~J} / \mathrm{g}$ for SD5. These results show that the method of solid dispersion preparation could generate a more amorphous state of MLX in solid dispersion samples (because of a lower melting point) and also produce a more homogenous matrix of drug/polymer compared to physical mixtures (because of a lower enthalpy of fusion). Similar results had been reported before for rofecoxib and poloxamer 188 (Shah et al., 2007; Barzegar-Jalali et al., 2012).

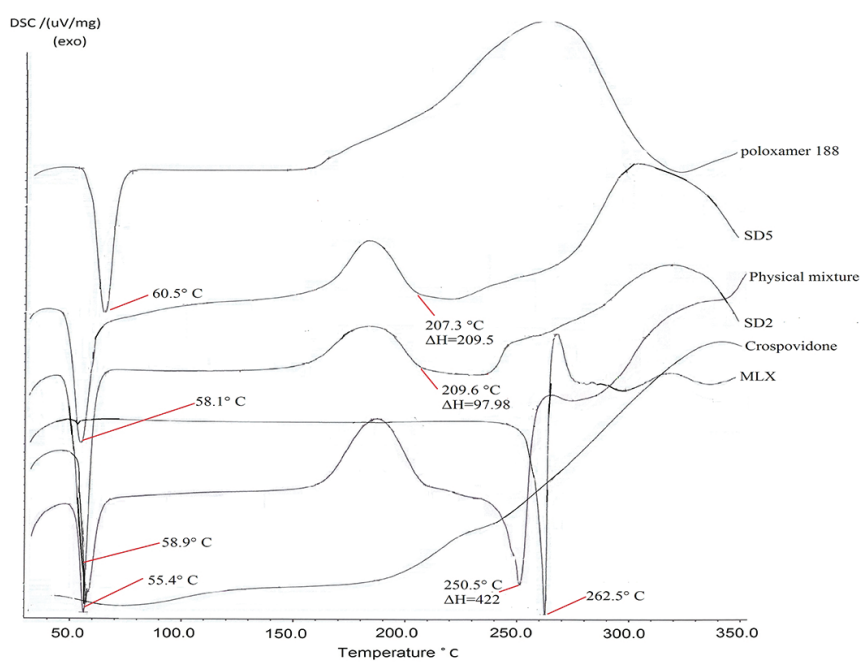

FIGURE 1 - The DSC thermograms of poloxamer 188, crospovidone, pure MLX, physical mixture of MLX and polymers and MLX SDs (SD2 and SD5).

\section{XRD studies}

The XRD pattern of pure MLX, crospovidone, poloxamer, MLX SDs (SD2 and SD5), and physical mixture are exhibited in Figure 2. X-ray diffraction of MLX showed sharp peaks at $2 \theta$ values of $13^{\circ}, 14.9^{\circ}, 18.5^{\circ}$ and $25.7^{\circ}$. The X-ray diffraction spectrum of crospovidone exhibited a halo pattern, indicating a completely amorphous state of these polymers. Moreover, the X-ray diffraction spectrum of poloxamer 188 showed characteristic peaks at $18.2^{\circ}$ and $25.1^{\circ}$. The intensity of the MLX characteristic peaks in the physical mixture of MLX and polymers reduced due to the polymer dilution effect, while a significant decrease in the intensity of characteristic peaks of MLX in MLX SDs, compared to pure MLX, suggested a significant reduction in MLX crystallinity due to MLX amorphization and its incorporation in the carrier structure (Barzegar-Jalali et al., 2012).

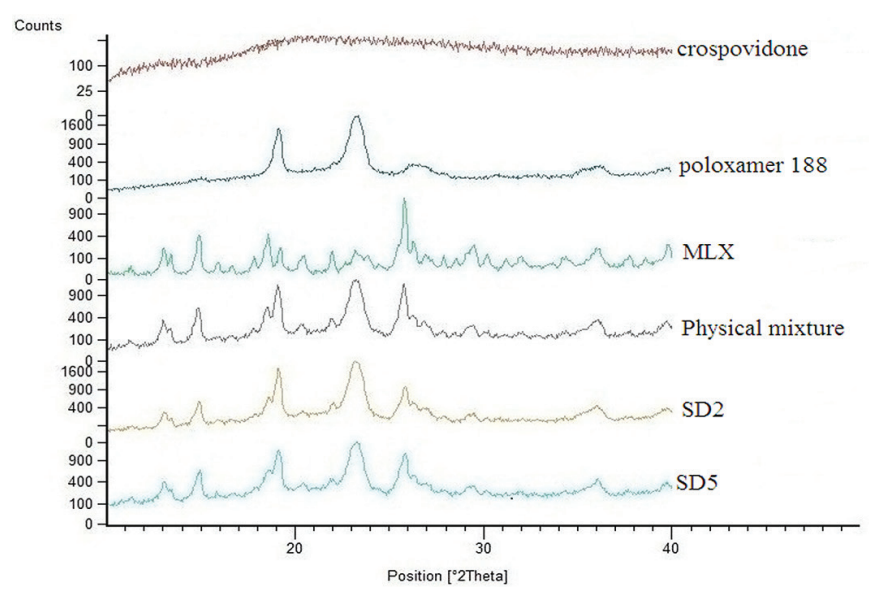

FIGURE 2 - XRD pattern of pure MLX, poloxamer 188, crospovidone, their physical mixture and MLX SDs (SD2 and SD5).

\section{Preparation and characterization of ODTs}

The optimized MLX SD, which showed the highest saturation solubility (SD2), was selected to develop MLX ODTs. The ODT compositions in this study are listed in Table III.

On a large scale, the flowability of powder blends could affect the content uniformity of tablets. Thus powder blends were evaluated for Hausner's ratio and Carr's index before tablet compression. Hausner's ratio and Carr's index of powder blends were less than 1.2 and 11 respectively, indicating a good flowability of powder blends (Shah, Tawakkul, Khan, 2008). MLX ODTs were prepared by the direct compression method because it is an easy and low-cost method (Medina, Kumar, 2006). 
TABLE III - Factorial design of MLX ODTs (F1 to F8) and the response parameters

\begin{tabular}{|c|c|c|c|c|c|c|c|c|}
\hline \multirow{2}{*}{$\begin{array}{l}\text { Ingredients } \\
\text { (mg/tablet) }\end{array}$} & \multicolumn{8}{|c|}{ Formula code } \\
\hline & $\mathrm{F} 1$ & $\mathrm{~F} 2$ & F3 & $\mathrm{F} 4$ & F5 & F6 & F7 & $\mathrm{F} 8$ \\
\hline $\begin{array}{l}\text { MLX-SD } \\
\text { (equivalent } \\
7.5 \mathrm{mg} \text { MLX) } \\
\text { (mg) }\end{array}$ & 24.3 & 24.3 & 24.3 & 24.3 & 24.3 & 24.3 & 24.3 & 24.3 \\
\hline $\begin{array}{l}\text { Mannitol } \\
(\mathrm{mg})\end{array}$ & 76.8 & 92.16 & 71.8 & 86.16 & 76.8 & 92.16 & 71.8 & 86.16 \\
\hline Avicel (mg) & 38.4 & 23.04 & 35.9 & 2154 & 38.4 & 23.04 & 35.9 & 21.54 \\
\hline $\begin{array}{l}\text { Mannitol/ } \\
\text { Avicel ratio }\end{array}$ & 2 & 4 & 2 & 4 & 2 & 4 & 2 & 4 \\
\hline $\begin{array}{l}\text { Crospovidone } \\
(\mathrm{mg})\end{array}$ & 7.5 & 7.5 & 15 & 15 & 7.5 & 7.5 & 15 & 15 \\
\hline $\begin{array}{l}\text { Aspartam } \\
(\mathrm{mg})\end{array}$ & 1.5 & 1.5 & 1.5 & 1.5 & 1.5 & 1.5 & 1.5 & 1.5 \\
\hline $\begin{array}{l}\text { Mg stearate } \\
\text { (mg) }\end{array}$ & 1.5 & 1.5 & 1.5 & 1.5 & 1.5 & 1.5 & 1.5 & 1.5 \\
\hline $\begin{array}{l}\text { Total weight } \\
(\mathrm{mg})\end{array}$ & 150 & 150 & 150 & 150 & 150 & 150 & 150 & 150 \\
\hline Force $(\mathrm{kN})$ & low & low & low & low & high & high & high & high \\
\hline Hardness & $23.25 \pm 5.8$ & $25.75 \pm 4$ & $22.42 \pm 5.5$ & $17.87 \pm 3.6$ & $44.87 \pm 4.3$ & $45.5 \pm 5.4$ & $45.75 \pm 6.1$ & $34.37 \pm 2.1$ \\
\hline $\begin{array}{l}\text { Disintegration } \\
\text { time }\end{array}$ & $18 \pm 13.8$ & $25 \pm 5$ & $13.33 \pm 1.1$ & $14.3 \pm 3.6$ & $31.6 \pm 4.3$ & $32.5 \pm 3.1$ & $12 \pm 2$ & $12.66 \pm 2.5$ \\
\hline $\begin{array}{l}\% \text { of } \\
\text { dissolved } \\
\text { meloxicam } \\
\text { after } 30 \mathrm{~min}\end{array}$ & $76.36 \pm 3.4$ & $79.33 \pm 4.9$ & $77.2 \pm 3.06$ & $80.4 \pm 1.6$ & $77.8 \pm 4.3$ & $80.16 \pm 2.5$ & $78.1 \pm 3.91$ & $82.66 \pm 5.1$ \\
\hline Friability\% & $1.34 \pm 0.08$ & $0.88 \pm 0.03$ & $1 \pm 0.054$ & $1.33 \pm 0.04$ & $0.87 \pm 0.06$ & $1.12 \pm 0.03$ & $0.7 \pm 0.09$ & $1.26 \pm 0.04$ \\
\hline
\end{tabular}

The average MLX content of the prepared MLX ODT formulations were in the range of $74.8 \pm 4.25-94.1 \pm 3.16 \%$ and met the United States Pharmacopeia (USP) content uniformity requirements (USP, 2007).

\section{Friability}

Tablet friability could reflect the tablet's physical strength. The acceptable friability range for ODTs was mentioned to be $0.95 \%$ to $1.5 \%$ (Mendes, Anaebonam 1990). The friability of conventional tablets must be less than $1 \%$ (USP, 2006). The friability of the prepared MLXODT formulations did not exceed $1.34 \pm 0.08 \%$ (Table III). Moreover, no tablet had cracked, split or broken in formulations.

\section{Disintegration time}

ODTs should disintegrate rapidly after placement in the mouth, thus the disintegration time is one of the most crucial characteristics of ODTs. Moreover, ODTs should have sufficient hardness and friability to withstand handling without substantial breakage and attrition. Ideally, ODTs showed a disintegration time of $60 \mathrm{~s}$ or less, and showed the hardness of at least $40 \mathrm{~N}$ (Ramtoola, Pabari, Kelly, 2008). However, increasing the hardness of tablets could increase the disintegration time and decrease the amount of dissolved drug after known time. One of the important aims of this study was the preparation of ODTs with hardness greater than $40 \mathrm{~N}$ without any significant effect on the disintegration time and the percentage of the dissolved MLX after $30 \mathrm{~min}$. Therefore, the crucial responses that were selected as dependent variables were disintegration time, hardness, and the percent of dissolved MLX in the dissolution media after $30 \mathrm{~min}$. The results obtained for the disintegration time of the prepared MLX-ODT tablets are summarized in Tables III. Six formulations 
showed a disintegration time of lower than $30 \mathrm{sec}$ and two formulations showed a disintegration time more than $30 \mathrm{sec}$. F6 showed the longest disintegration time $(32.5 \pm 3.1 \mathrm{sec})$ and F7 showed the shortest disintegration time $(12 \pm 2 \mathrm{sec})$. An ANOVA analysis showed that only one factor, namely, percent of superdisintegrant (C) had significant effects on the disintegration time of the prepared MLX ODTs at $\mathrm{p}<0.0001$ (Figure 3). The results show that the disintegration time decreased as the percentage of superdisintegrant increased. However, the mannitol-avicel ratio and compression force did not have significant effects on the disintegration time. The resulting equation for responses, Y1 (disintegration time), is presented below:

Disintegration time $=+40.58250-2.75850 *$ percent of superdisintegrant.

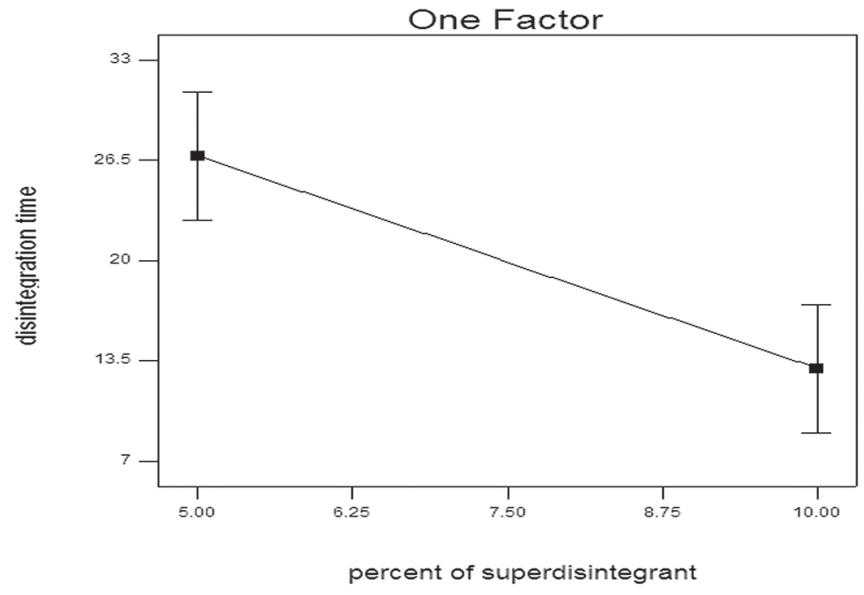

FIGURE 3 - Effect of percent of superdisintegrant on the disintegration time of MLX ODT.

\section{Hardness of MLX ODTs}

The hardness of the prepared MLX-ODTs was in the range of $17.87-45.75 \mathrm{~N}$ (Table III) with the highest hardness values observed in F5, F6, F7, and F8 with the mean hardness values of $44.87 \pm 4.3,45.5 \pm 5.4,45.75 \pm 6.1$, $34.37 \pm 2.16 \mathrm{~N}$ respectively. An ANOVA analysis showed that only one factor, namely, compression force $(\mathrm{A} ; \mathrm{p}<$ 0.0007), had a significant effect on the hardness of the prepared MLX-ODTs (Figure 4). The results show that the hardness of tablets increased as the compression force was increased. Other factors (the percentage of superdisintegrant and mannitol-avicel ratio) did not have a significant effect on the hardness of MLX ODTs. The resulting equation for responses, Y2 (hardness), is presented below:

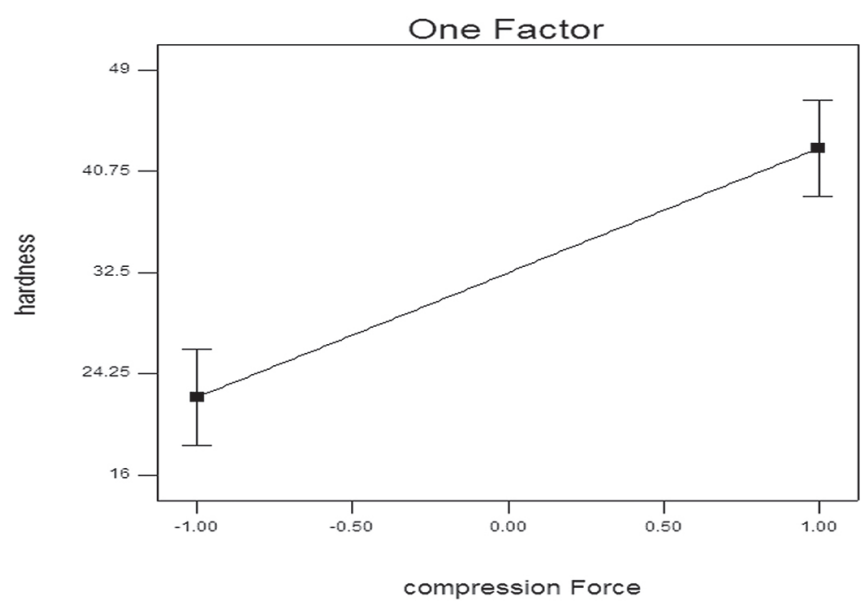

FIGURE 4 - Effect of compression force on the hardness of MLX ODT.

Hardness $=+32.47250+10.15000 *$ compression Force

\section{In vitro dissolution studies}

Figure 5 represents the dissolution profiles of the prepared MLX ODTs. According to the United States Pharmacopeia (USP), an MLX tablet should release 70 percent of its drug content after $30 \mathrm{~min}$. F4 and F8 showed the highest percentage of MLX release after 30 min; $80.4 \pm 1.6$ and $82.66 \pm 5.1$ respectively. This high amount of release could be due to the high amount of water-soluble mannitol and low amount of low watersoluble avicel in their formulations. The use of a high amount of mannitol in tablet formulation could help the dissolution of poorly water-soluble drugs (Myers, Battist, Fuisz, 1995). Moreover, F4 and F8 released about 50\% of their drug content only after $2 \mathrm{~min}$. This burst release of MLX might be due to the effect of water-soluble mannitol, which could increase the degree of cracking and bursting (Shirai et al., 1994). An ANOVA analysis showed only one factor, namely, mannitol-avicel ratio $(p<0.0001)$ had a significant effect on the percentage of dissolved MLX (after $30 \mathrm{~min}$ ) from the prepared MLX ODTs (Figure 6). The results also showed that the percentage of MLX dissolved after $30 \mathrm{~min}$ increased as the mannitol-avicel ratio become greater. The compression force and percentage of super disintegrant did not show any significant effects on the percent of MLX released after $30 \mathrm{~min}$. The resulting equation for responses, Y3 (percent of MLX that dissolved after 30 min), is presented below:

Percent of dissolved meloxicam after $30 \mathrm{~min}$ $=+74.09250+1.63625 *$ mannitol - avicel ratio 


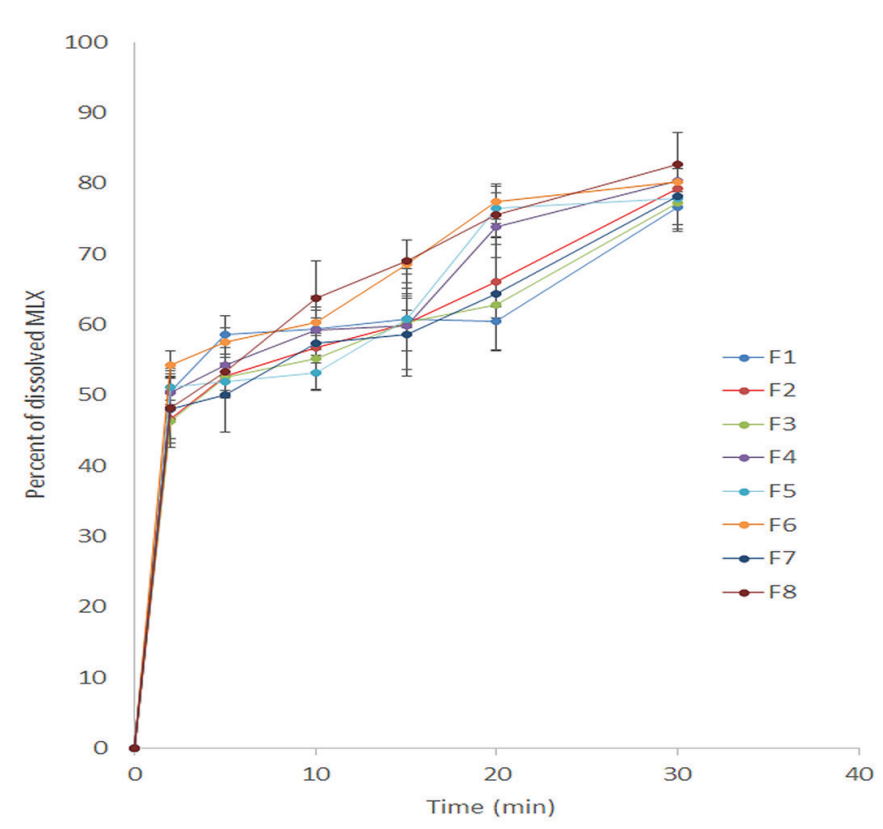

FIGURE 5 - The dissolution profiles of the prepared MLX ODTs.

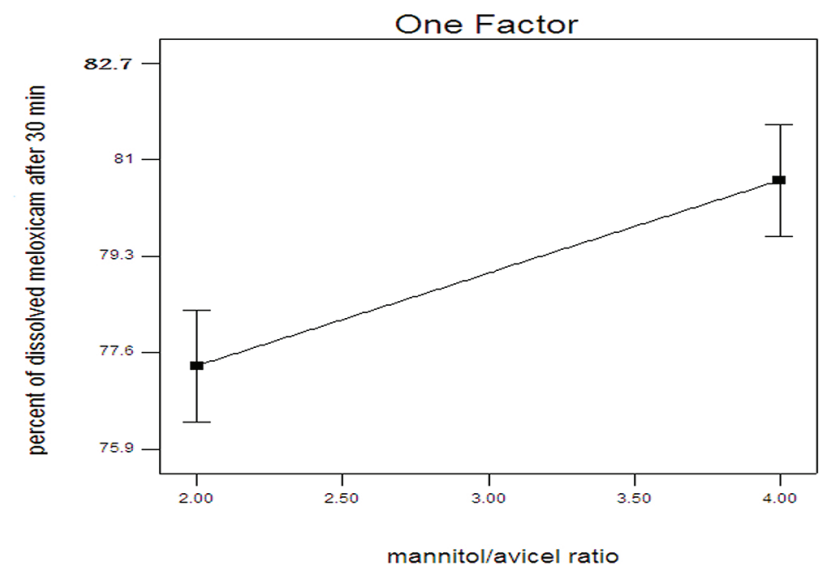

FIGURE 6 - The effect of mannitol/avicel ratio on the percent of dissolved meloxicam that dissolved after $30 \mathrm{~min}$ in the dissolution medium.

\section{Optimum ODTs formulation}

Based on the responses, the software was used to perform a hot spot analysis to obtain the optimum formulation variables (compression force, percent of superdisintegrant and mannitol/avicel ratio) to produce ODTs with the desired characteristics. The request was to minimize the disintegration time, and maximize the hardness and percent of released MLX from the formulation after $30 \mathrm{~min}$. Therefore; the $\mathrm{F} 8$ formulation was determined to be the optimal formulation.

\section{CONCLUSION}

The main goal of a solid dispersion drug delivery system is to improve the water solubility of poorly watersoluble drugs. Solid dispersion prepared by the melting method with crospovidone and poloxamer 188 with special physicochemical properties have shown to be one of the most attractive approaches to enhance the solubility of the poorly water-soluble drug meloxicam. On the other hand, orally disintegrating tablets prepared from solid dispersion represent a promising approach to improving the solubility and slow onset of action of drugs, especially for BCS Class II of drugs, including meloxicam with poor dissolution properties. The present study demonstrated an effective method for the preparation of a suitable dosage form of meloxicam with improved solubility and onset of action. This dosage form could be suitable for administration in elderly patients with dysphagia.

\section{REFERENCES}

Ahmed M, Khanna D, Furst DE. Meloxicam in rheumatoid arthritis. Expert Opin Drug Metab. 2005;1(4):739-751.

Aoki T, Yamaguchi H, Naito H, Shiiki K, Izawa K, Ota Y, et al. Premedication with cyclooxygenase-2 inhibitor meloxicam reduced postoperative pain in patients after oral surgery. Int $\mathrm{J}$ Oral Maxillofac Surg. 2006;35(7):613-617.

Barzegar-Jalali M, Alaei-Beirami M, Javadzadeh Y, Mohammadi G, Hamidi A, Andalib S, et al. Comparison of physicochemical characteristics and drug release of diclofenac sodium-eudragit $\mathbb{}$ RS100 nanoparticles and solid dispersions. Powder Technol. 2012;219:211-216.

Budavari S,O'Neil MJ, Smith A, Heckelman PE, Obenchain Jr JR, Gallipeau JAR, D'Arecea MR. The Merck Index, an Encyclopedia of Chemicals Drugs and Biologicals. $13^{\text {rd }}$ ed. Whitehouse Station, NJ: Merck \& Co; 2001.

Chiou WL, Riegelman S. Pharmaceutical applications of solid dispersion systems. J Pharm Sci. 1971;60(9):1281-1302.

Distel M, Mueller C, Bluhmki E, Fries J. Safety of meloxicam: a global analysis of clinical trials. Rheumatology. 1996;35(Suppl 1):68-77.

Fujii M, Okada H, Shibata Y, Teramachi H, Kondoh M, Watanabe Y. Preparation, characterization, and tableting of a solid dispersion of indomethacin with crospovidone. Int J Pharm. 2005;293(1):145-153. 
Ghorab MM, Abdel-Salam HM, El-Sayad MA, Mekhel MM. Tablet formulation containing meloxicam and $\beta$-cyclodextrin: mechanical characterization and bioavailability evaluation. AAPS Pharm Sci Tech. 2004;5(4):63-68.

He CX, He ZG, Gao JQ. Microemulsions as drug delivery systems to improve the solubility and the bioavailability of poorly water-soluble drugs. Expert Opin Drug Deliv. 2010;7(4):445-460.

Jahangiri A, Barzegar-Jalali M, Garjani A, Javadzadeh Y, Hamishehkar H, Rameshrad M, et al. Physicochemical characterization and pharmacological evaluation of ezetimibePVP K30 solid dispersions in hyperlipidemic rats. Colloids Surf B Biointerfaces. 2015;134:423-430.

Jung JY, Yoo SD, Lee SH, Kim KH, Yoon DS, Lee KH. Enhanced solubility and dissolution rate of itraconazole by a solid dispersion technique. Int J Pharm. 1999;187(2):209-218.

Leuner C, Dressman J. Improving drug solubility for oral delivery using solid dispersions. Eur J Pharm Biopharm. 2000;50(1):47-60.

Lieberman HA, Lachman L. Pharmaceutical dosage forms tablets. 2 ed. New York: Marcel Dekker; 1989. 3 v.

Medina MD, Kumar V. Evaluation of cellulose II powders as a potential multifunctional excipient in tablet formulations. Int $\mathbf{J}$ Pharm. 2006;322(1):31-35.

Mendes, RW, Anaebonam AO. Chewable tablet. In: Swarbrick J, Boylan JC (editors). Encyclopedia of pharmaceutical technology New York: Marcel Dekker; 1990. v. 2, p. 397.

Merisko-Liversidge E, Liversidge GG, Cooper ER. Nanosizing: a formulation approach for poorly-water-soluble compounds. Eur J Pharm Sci. 2003;18(2):113-120.

Myers GL, Battist GE, Fuisz RC. Process and apparatus for making rapidly dissolving dosage units and products therefrom. Patent No. WO 1995034293-A1. Fuisz Technologies; 1995.

Pairet M, Van Ryn J, Schierok H, Mauz A, Trummlitz G, Engelhardt G. Differential inhibition of cyclooxygenases-1 and2 by meloxicam and its 4'-isomer. Inflamm Res. 1998;47(6):270276.

Punčochová K, Heng JY, Beránek J, Štěpánek F. Investigation of drug-polymer interaction in solid dispersions by vapour sorption methods. Int J Pharm. 2014;469(1):159-167.
Ramtoola Z, Pabari R, Kelly J. Method of producing fast dissolving tablets. United States patent application US 12/594,401. Royal College of Surgeons in Ireland; 2008.

Rauck RL, Tark M, Reyes E, Hayes TG, Bartkowiak AJ, Hassman D, et al. Efficacy and long-term tolerability of sublingual fentanyl orally disintegrating tablet in the treatment of breakthrough cancer pain. Curr Med Res Opin. 2009;25(12):2877-2885.

Serajuddin A. Solid dispersion of poorly water-soluble drugs: Early promises, subsequent problems, and recent breakthroughs. J Pharm Sci. 1999;88(10):1058-1066.

Serajuddin AT. Salt formation to improve drug solubility. Adv Drug Deliv Rev. 2007;59(7):603-616.

Shah TJ, Amin AF, Parikh JR, Parikh RH. Process optimization and characterization of poloxamer solid dispersions of a poorly water-soluble drug. AAPS PharmSciTech. 2007;8(2):E18-24.

Shah RB, Tawakkul MA, Khan MA. Comparative evaluation of flow for pharmaceutical powders and granules. AAPS Pharmscitech. 2008;9(1):250-258.

Shen YC, Lee MY, Lin CC, Chen CH. Orally disintegrating olanzapine for the treatment of a manic patient with esophageal stricture plus chronic pharyngitis. Prog Neuropsychopharmacol Biol Psychiatry 2007;31(2):541-242.

Shibata Y, Fujii M, Sugamura Y, Yoshikawa R, Fujimoto S, Nakanishi S, Motosugi Y, Koizumi N, Yamada M, Ouchi K, Watanabe Y. The preparation of a solid dispersion powder of indomethacin with crospovidone using a twin-screw extruder or kneader. Int J Pharm. 2009;365(1):53-60.

Shirai Y, Sogo K, Fujioka H, Nakamura Y. Role of low substituted hydroxypropyl cellulose in dissolution and bioavailability of novel fine granule system for masking bitter taste. Biol Pharm Bull.1994;17:427-431.

United States Pharmacopeia. USP. United States Pharmacopeia and National Formulary (USP 29-NF 24). Rockville MD: United States Pharmacopeia Convention; 2006. v. 2, p. 3046.

United States Pharmacopeia. USP. United States Pharmacopeia and National Formulary (USP 30-NF 25). Rockville MD: United States Pharmacopeia Convention; 2007. v. 14, p. 1553-1554.

Received for publication on $09^{\text {th }}$ November 2016 Accepted for publication on $05^{\text {th }}$ July 2017 